\title{
Efficient height measurements in single images based on the detection of vanishing points
}

\author{
Fernanda A. Andalóa,*, Gabriel Taubin ${ }^{\mathrm{b}}$, Siome Goldenstein ${ }^{\mathrm{c}}$ \\ ${ }^{a}$ SAMSUNG Research Institute Brazil (SRBR), \\ CEP 13097-160, Campinas, SP, Brazil \\ ${ }^{b}$ School of Engineering, Brown University, \\ Providence, RI 02912 USA \\ ${ }^{c}$ Institute of Computing, University of Campinas (Unicamp), \\ CEP 13083-852, Campinas, SP, Brazil
}

\begin{abstract}
Surveillance cameras have become a customary security equipment in buildings and streets worldwide. It is up to the field of Computational Forensics to provide automated methods for extracting and analyzing relevant image data captured by such equipment. In this article, we describe an effective and semi-automated method for detecting vanishing points, with their subsequent application to the problem of computing heights in single images. With no necessary camera calibration, our method iteratively clusters segments in the bi-dimensional projective space, identifying all vanishing points - finite and infinite - in an image. We conduct experiments on images of man-made environments to evaluate the output of the proposed method and we also consider its application on a photogrammetry framework.
\end{abstract}

Keywords: Photogrammetry, Vanishing point, Height measurement, Projective space

\section{Introduction}

By analyzing certain image properties - known object size, occlusion, stereoscopic vision, focus, gradient, texture, vergence - we can infer three-dimensional

\footnotetext{
* Corresponding author

Email addresses: f.andalo@samsung.com (Fernanda A. Andaló), taubin@brown.edu (Gabriel Taubin), siome@ic.unicamp.br (Siome Goldenstein)

Preprint submitted to Computer Vision and Image Understanding

January 4, 2015
}

(C) 2015. This manuscript version is made available under the Elsevier user license http://www.elsevier.com/open-access/userlicense/1.0/ 
(3D) information of the geometry portrayed in the images. This inferred 3D data 5 can be employed in various computer vision applications: image-based rendering [1, 2], automated driving [3], object detection 4], and forensic science [5].

The process of extracting, from images, geometric properties, such as heights, areas, and angles, is denominated photogrammetry. Photogrammetry methods are widely used in forensic investigations, where it can help corroborate pieces of evidence [5]. Analysis of car accidents and of human height are the two main examples of forensic activities that involve photogrammetry [5].

Even in low quality images, like the ones captured by surveillance cameras, with the aid of photogrammetry we can estimate the height of objects and people, useful identification characteristic when the face of the suspects cannot be identified or the details in their clothes are not relevant. Conversely, the suspects are often not on the crime scene at the time of the investigation, or the scene has changed. In these cases, the image itself is the only source of information.

When only one image depicting the scene is available, the essential problem 20 is the recovery of the third dimension, given that this information was not captured in the acquisition process, in which the 3D scene was projected onto the $2 \mathrm{D}$ image plane. In particular, perspective distortions also occur. For example, objects that are away from the camera appear smaller in the image than objects that are closer.

To solve this problem, the first photogrammetrists assumed some a priori information: internal camera parameters - focal length, optical center, scale, distortions, and skew factor -, and the camera position in relation to the scene. However, this is only valid when the accuracy of these values is high, since any deviation can generate large measurement errors $[\underline{6}$.

Recent works on photogrammetry can be generally classified into two categories: the ones that exploit 3D information from multiple images and the ones that analyze geometric properties in a single image. The first category deals with 3D reconstruction of the scene, from multiple views, to estimate homography or to calibrate the camera [7, 8, 9]. Single-view based methods can only 
rely on the analysis of geometric properties [10, 11, 12, 13] but, in this scenario, they often use previously positioned markers for calibration purposes [12, 11]. Although these methods attain high accuracy, they cannot be used in forensic applications, where often a previously taken image is the only source of information.

40 Here we propose a single-view method that detects vanishing points - invariant geometric features that can aid photogrammetry. A vanishing point can be defined as an image point where the projection of a set of real-world parallel lines converges, assuming perspective projection. A vanishing line is a line that contains two vanishing points.

The credit for discovering linear perspective is given to renaissance artist Leon Battista Alberti and architect Filippo Brunelleschi [14. Alberti's treatise, De pictura [15, published in 1435, encloses the first scientific study of perspective. By the 1470s, several artists were able to produce their works of art demonstrating a full understanding of the principles of linear perspective.

Leonardo da Vinci, beginning in 1481, also studied and employed perspective in his earlier paintings (Figure 1).

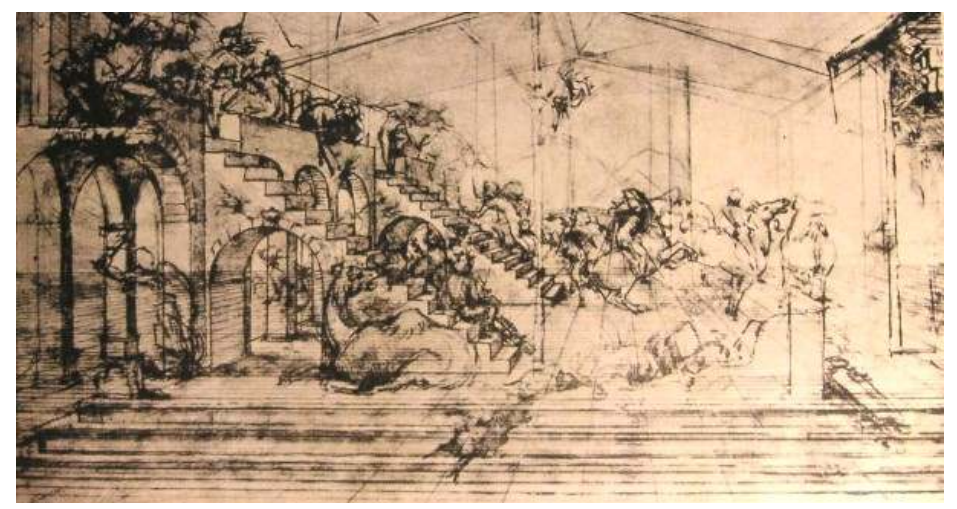

Figure 1: Study for the painting Adoration of Magi, by Leonardo da Vinci showing the use of perspective.

In this article, we use a geometric approach to effectively estimate the location of vanishing points - finite and infinite ones - in images of urban and 
indoor spaces, with the Manhattan-world assumption. We are assuming that the scene has a natural cartesian 3D coordinate system, which is plausible for indoor, outdoor city, and even some country scenes [16].

By representing image segments in the bi-dimensional projective space $\mathbb{R P}^{2}$, our method clusters them into groups of segments that converge to a unique vanishing point locality. The detected features are then used to identify the ground plane and the vertical direction of the scene. This information is finally inserted into a photogrammetry algorithm proposed by Criminisi [10, with the ultimate goal of measuring the height of objects and people in single images.

This article starts by presenting, in Section 2 , a background on the measurement of heights in single images based on vanishing points. In Section 3 , we present our vanishing point detector. In Section 4 , we show how to estimate the scene vertical direction and also how to detect the ground plane vanishing line. The experiments and their results are provided in Section 5. Finally, Section 6 states the conclusions of this work.

\section{Background}

The projection of a world point $X \in \mathbb{R}^{3}$ into an image point $x \in \mathbb{R}^{2}$, considering perspective projection, is described by projection matrix $\mathrm{P} \in \mathbb{R}^{3 \times 4}$ as

$$
\tilde{x}=\mathrm{P} \tilde{X}=\mathrm{K}[\mathrm{R} \mid \mathrm{T}] \tilde{X}=\left[\begin{array}{lll}
p_{1} & p_{2} & p_{3} \\
p_{4}
\end{array}\right] \tilde{X},
$$

70 $\mathrm{K}$ is the matrix representing the intrinsic parameters of the camera; the extrinsic parameters are $\mathrm{R}$ - rotation matrix - and $\mathrm{T}$ - translation vector from the world to the camera system; $p_{1}, p_{2}, p_{3}$ and $p_{4}$ are the columns of $\mathrm{P}$; and the equality is up to scale.

75

In [17, the authors prove that $p_{1}, p_{2}$ and $p_{3}$ are the orthogonal vanishing points corresponding to the world coordinate system, and that $p_{4}$ is the image of the world origin. Here we denote them as $v_{x}, v_{y}, v_{z}$. 
Considering that $v_{x}$ and $v_{y}$ are the two vanishing points on the vanishing line, we can say that $p_{4}$ must not be on the same line. If it does, then $v_{x}$, $v_{y}$, and $p_{4}$ are linearly dependent. Hence the fourth column can be set to $p_{4}=m /\|m\|=\bar{m}$, where $m$ is the vanishing line [6]. The final projection matrix is

$$
\mathrm{P}=\left[\begin{array}{llll}
v_{x} & v_{y} & \alpha v_{z} \bar{m}
\end{array}\right],
$$

where $\alpha$ is an unknown scalar referred as metric factor. If $v_{z}$ and $m$ are available, then the metric factor $\alpha$ is the only unknown value.

To measure heights in images, we must compute the distance between points in two different planes. Let $v_{z}$ be the vanishing point that indicates the scene vertical direction, and $m$ the ground vanishing line. Considering the projection matrix P (Equation 2), Criminisi [18] proved that for an arbitrary object $\lambda$, with height $Z_{\lambda}$ and delimited by image points $t_{\lambda}$ and $b_{\lambda}$ (top and bottom points), it holds that

$$
\alpha Z_{\lambda}=-\frac{\left\|b_{\lambda} \times t_{\lambda}\right\|}{\left(\bar{m} \cdot b_{\lambda}\right)\left\|v_{z} \times t_{\lambda}\right\|} .
$$

Figure2 2 illustrates Equation 3 . Point $o_{\lambda}$ represents the intersection between vanishing line $m$ and the line that contains $t_{\lambda}$ and $b_{\lambda}$. This intersection point helps to define a ratio of distances between planes and, using this value, one can compute $\frac{Z_{\lambda}}{Z_{c}}$, where $Z_{c}$ is the camera's distance. However, it is simpler to compute $Z_{\lambda}$ via a reference measurement in the image with a known length [6].

Thus, if we want to measure height $Z_{o b j}$ of an object $o b j$, and $Z_{r e f}$ is a known distance on the same image, i.e., it is a reference distance between points $t_{r e f}$ and $b_{r e f}$, then Equation 3 allows the computation of the scale factor $\alpha$ and subsequently the distance $Z_{o b j}$ between $t_{o b j}$ and $b_{o b j}$. The following steps can be used to compute height $Z_{o b j}$ :

1. Detect vanishing points in $I$.

2. Identify vanishing point $v_{z}$ associated with the scene vertical direction.

3. Estimate vanishing line $m$ associated with the ground plane. 


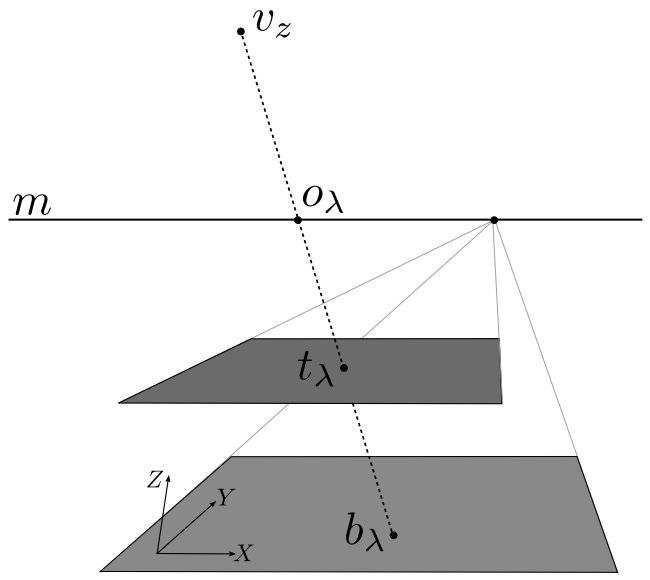

Figure 2: Distance between the planes that contain points $t_{\lambda}$ and $b_{\lambda}$. Point $o_{\lambda}$ represents the intersection between vanishing line $m$ and line that contains points $t_{\lambda}$ and $b_{\lambda}$.

4. Compute scale factor $\alpha$ by applying Equation 3 with object ref, i.e., $\lambda=r e f$.

5. Compute $Z_{o b j}$ by applying Equation 3 with object $o b j$, i.e., $\lambda=o b j$, and $\alpha$.

The process of computing heights in images is illustrated in Figure 3 According to Criminisi [10], efficient measurements in images can be done by accurately detecting the vanishing points, and estimating vertical vanishing point $v_{z}$ and vanishing line $m$ that represents the ground plane (steps 1, 2 and 3). Therefore, we can use the output of our detector to estimate $v_{z}$ and $m$.

In the subsequent sections, we describe our method to detect vanishing points and how to estimate, from them, vertical vanishing point $v_{z}$, and ground vanishing line, $m$.

\section{Estimating vanishing points in single images}

The problem of locating vanishing points in 2D perspective projection images has been studied since the 80 s. This task is generally seen as the computation of line intersections, but due to quantization errors, lines that correspond to a single vanishing point intersect inside an area denominated vanishing region. 


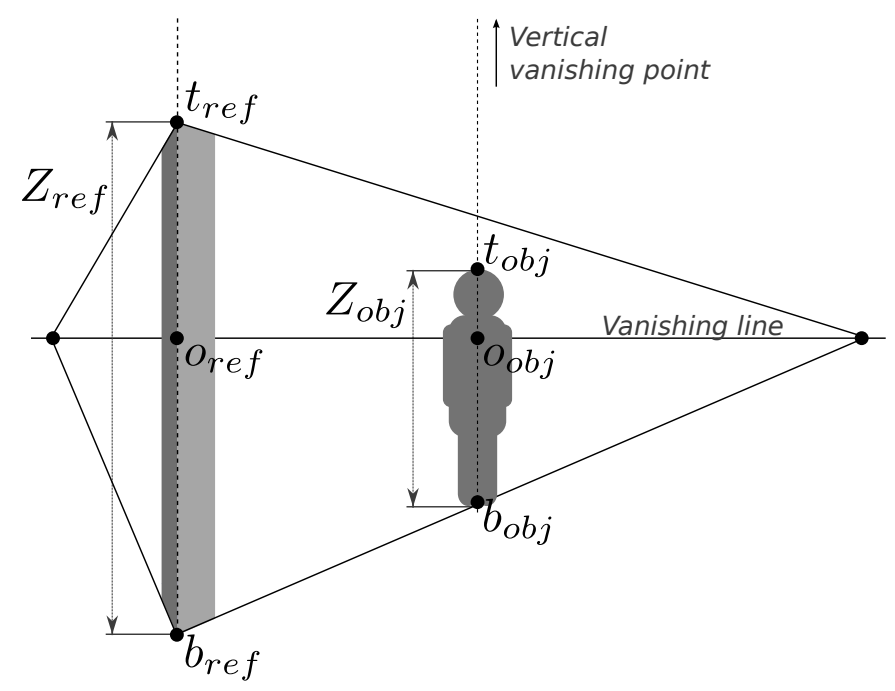

Figure 3: Measuring the height of a real object depicted in an image. Height $Z_{o b j}$ can be estimated with the aid of known height $Z_{\text {ref }}$ of a reference object.

To confront the vanishing region problem, methods often divide the vanishing point detection in three main stages: detection of segments in the image, clustering of the these segments in groups that converge to a vanishing point, and vanishing point estimation for each cluster.

The first stage can be accomplished using an edge detector subsequently grouping the edges to form segments, e.g., Canny operator [19] and Hough transform [20. Methods can perform the other stages in two ways: using accumulator spaces [21, 22, 23, 24, 25] or using the image plane directly [26, 27].

Since Barnard's seminal work on the detection of vanishing points [21], methods have been employing different Hough transform techniques in quantized Gaussian spheres [22]. The problem in such methods are the artifacts often present in digital images, producing erroneous maxima on the quantized space [23].

The methods that use the image plane directly do not need accumulator techniques [26, 27. In this case, the accuracy of the vanishing point location is not limited by the space and the distances are preserved. However, it may be 
necessary to incorporate additional criteria to work with the infinite vanishing points.

Against these works, our method uses the bi-dimensional projective space $\mathbb{R P}^{2}$, or projective plane, transformed directly from the image space, to cluster the segments and detect all vanishing points, including the infinite ones, without additional criteria. Moreover, the space is not bounded, meaning it does no limit the location accuracy.

The proposed detector also has three stages:

1. Extracting line segments from the input image.

2. Clustering of the segments that converge to the same vanishing point (repeated until convergence):

\subsection{Selection of seeds.}

2.2. Grouping of segments based on the seeds and the intersection points.

3. Detection of a vanishing point for each final cluster.

Figure 4 illustrates the three steps to estimate the vanishing points, numerated as shown above. Each one of the steps is described in a subsection.

\subsection{Line segment detection}

We start by extracting line segments from the input image using a method proposed by Desolneaux et al. [28, 29]. They deal with the extracting problem by exploring the Helmholtz Principle [29]. Besides line segments, their method also produce, for each segment, its number of false alarms, which will be used to compute the quality of this segment.

The Helmholtz principle declares that if, in an image, the expectation of a certain observed configuration is small, then this grouping is a Gestalt, i.e, it is meaningful [29].

Definition 1 ( $\epsilon$-meaningful configuration). A configuration is $\epsilon$-meaningful if it occurs in an image in a number less than $\epsilon$. 


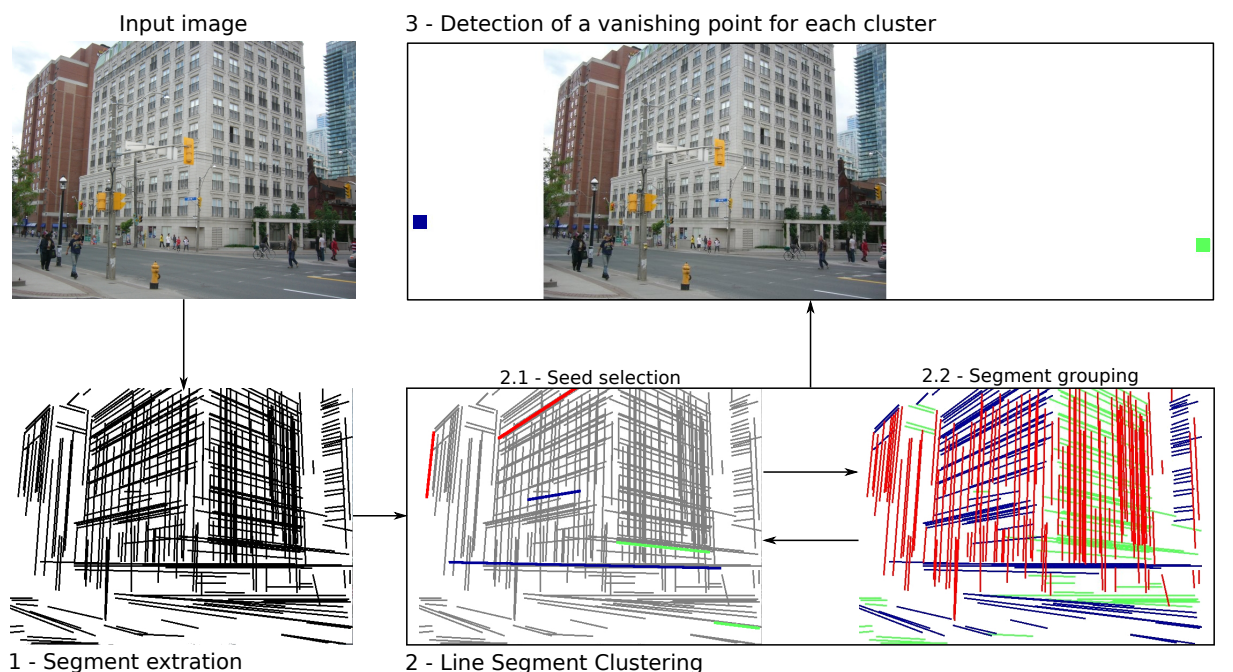

Figure 4: Illustration of the three steps to detect vanishing points in an image. Each color (red, green and blue) represents a different cluster. In step 3, the vertical vanishing point is not shown, because it lies at inifinity.

Let $f$ be a $N \times N$ image, and $A \in f$ a segment formed by a set of pixels $\left\{x_{i}\right\}, i=1, \ldots, l$. Consider a random variable $X_{i}$ where $X_{i}=1$ if the direction of pixe ${ }^{1} x_{i}$ is aligned to the direction of $A$, and $X_{i}=0$ otherwise. Therefore, $X_{i}$ has the following distribution with precision level $p$ :

$$
P\left[X_{i}=1\right]=p \quad \text { and } \quad P\left[X_{i}=0\right]=1-p .
$$

The direction of $x_{i}$ is aligned to the direction of $A$ with precision $p$ when

$$
D_{\text {ang }}\left(\operatorname{dir}\left(x_{i}\right), \operatorname{dir}(A)\right) \leq \pi p
$$
outputs the smallest angle between the two directions.

The number of aligned pixels in segment $A$ is represented by random variable $S_{l}=X_{1}+X_{2}+\ldots+X_{l}$. Because random variables $X_{i}$ are independent, $S_{l}$ has

\footnotetext{
${ }^{1}$ The direction of a pixel is defined by Desolneux et al. [29] as the direction orthogonal to the direction of the gradient at the pixel.
} 
the following binomial distribution:

$$
P\left[S_{l}=k\right]=\left(\begin{array}{l}
l \\
k
\end{array}\right) p^{k}(1-p)^{l-k} .
$$

To consider segment $A$ as a primitive for our method, we need to know if $A$ is $\epsilon$-meaningful among all segments in $f$.

Definition 2 ( $\epsilon$-meaningful segment). A l-length segment $A$ is $\epsilon$-meaningful if it contains a minimum of $k(l)$ aligned pixels, where

$$
k(l)=\min \left\{k \in \mathbb{N}, P\left[S_{l} \geq k\right] \leq \frac{\epsilon}{N^{4}}\right\} .
$$

The value $N^{4}$ is the number of oriented segments (defined by their initial and end pixels) in a $N \times N$ image.

Consider the $i$-th segment, with length $l_{i}$, and the event $e_{i}$ meaning "the $i$-th segment is $\epsilon$-meaningful". Let $\chi_{e_{i}}$ denote the characteristic function of this event, so that

$$
P\left[\chi_{e_{i}}=1\right]=P\left[S_{l_{i}} \geq k\left(l_{i}\right)\right]=\sum_{k=k\left(l_{i}\right)}^{l_{i}}\left(\begin{array}{c}
l_{i} \\
k
\end{array}\right) p^{k}(1-p)^{l_{i}-k} .
$$

Then the total of $\epsilon$-meaningful segments is represented by variable $R=$ $\chi_{e_{1}}+\chi_{e_{2}}+\ldots+\chi_{e_{N^{4}}}$, and expectation $E(R)$ relates to the number of false alarms.

Definition 3 (number of false alarms in a segment). Considering a $l_{0}$-length segment $A$ with at least $k_{0}$ aligned pixels, the number of false alarms of $A$ is

$$
F\left(k_{0}, l_{0}\right)=N^{4} P\left[S_{l_{0}} \geq k_{0}\right]=N^{4} \sum_{k=k_{0}}^{l_{0}}\left(\begin{array}{l}
l_{0} \\
k
\end{array}\right) p^{k}(1-p)^{l_{0}-k} .
$$

If we take into account all $\epsilon$-meaningful segments as primitives for our 165 method, we will have several low quality segments. To avoid these equivocal segments, we finally take into account only some of the $\epsilon$-meaningful segments, the maximal ones.

Definition 4 (Maximal segment). A segment $A$ is maximal if: 
$\begin{aligned} & \text { 1. } \forall B, B \subset A \Rightarrow F(B) \geq F(A) . \\ & \text { 170 } \text { 2. } \forall B, B \supset A \Rightarrow F(B)>F(A) .\end{aligned}$

The two parameters of the method by Desolneux et al. [29], threshold $\epsilon$ and precision level $p$, are not critical. Values $\epsilon=1$ and $p=\frac{1}{16}$ work well for all images [24].

We group the detected maximal segments in a set $\mathcal{S}=\left\{s_{1}, \ldots, s_{|S|}\right\}$, and each maximal segment $s_{i} \in \mathcal{S}$ has a number of false alarms denoted by $F_{i}$.

Consider the end points $\left(x_{1}, y_{1}\right)$ and $\left(x_{2}, y_{2}\right)$, in the image plane $\mathbb{R}^{2}$, that specify an arbitrary maximal segment. If we represent these points in $\mathbb{R P}^{2}$, we get $\left(x_{1}, y_{1}, 1\right)$ and $\left(x_{2}, y_{2}, 1\right)$, respectively, using homogeneous coordinates. Here we are also interested in the lines that correspond to the maximal segments, $\left(x_{1}, y_{1}, 1\right) \times\left(x_{2}, y_{2}, 1\right)$. So, for each maximal segment $s_{i}$, we compute the correspondent line as shown above, resulting in $l_{i}$. The computed lines form a set $\mathcal{L}=\left\{l_{1}, \ldots, l_{|S|}\right\}$.

\subsection{Segment clustering}

The clustering process consists in assigning each segment $s_{i} \in \mathcal{S}$ to a cluster $h$. Each cluster $h$ is a set formed by line segments assigned to it.

The clustering process has three main steps: determination of the first seeds, assignment of segments to clusters, and update of the seeds.

\subsubsection{First seeds}

The selection of the first seeds is done by considering a quality value for segments. The quality value $q_{i}$ of segment $s_{i}$ is defined as

$$
q_{i}=1-\left(\frac{F_{i}-\min F}{\max F-\min F}\right),
$$

where $\max F$ and $\min F$ are the maximum and minimum numbers of false alarms among segments in $\mathcal{S}$, respectively. Note that the quality value of a segment is inversely proportional to its number of false alarms, i.e., the lower the number of false alarms, higher its quality. 
The number of clusters, $H$, is chosen by the user. The $2 H$ higher quality segments are randomly selected, in pairs, to be the seeds for the $H$ clusters. The two segment seeds of cluster $h$ are denoted by $\alpha_{h}$ and $\beta_{h}$.

The method converges in fewer steps when the first seeds are selected based on the quality of the segments. If random segments are chosen as the first seeds instead, the method yields the same results, except that it takes more steps.

\subsubsection{Assignment step}

200

The assignment step is responsible for selecting a cluster for each segment in $\mathcal{S}$. This is done by computing the distance between pseudo-centroids $c_{h}=$ $l_{\alpha_{h}} \times l_{\beta_{h}}$, defined for each cluster $h=1, \ldots, H$, and lines in $\mathcal{L}$.

The distance between an arbitrary point $c$ and an arbitrary line $l$ in $\mathbb{R P}^{2}$ can be computed as

$$
D_{\text {proj }}(c, l)=\frac{|c \cdot l|}{\|c\|\|l\|} .
$$

The formula for distance $D_{\text {proj }}$ is obtained by considering the angle between the line that corresponds to $c$ and the plane that corresponds to $l$ in $\mathbb{R P}^{3}$.

Distance $D_{\text {proj }}$ only measures the symmetry between points and lines, because it is not a complete metric. Note that $D_{\text {proj }}$ can be used arbitrarily also between two points in $\mathbb{R P}^{2}$.

The cluster assigned to line segment $s_{i}$ is the one with the closest pseudocentroid., i.e,

$$
\operatorname{cluster}\left(s_{i}\right)=\underset{j \in[1, H]}{\operatorname{argmin}} D_{\text {proj }}\left(c_{j}, l_{i}\right) .
$$

\subsubsection{Update step}

In this step, the method selects new seeds $\alpha_{h}$ and $\beta_{h}$ for each cluster $h=$ $210 \quad 1, \ldots, H$.

New seed $\alpha_{h}$ is chosen as the segment in cluster $h$ with closest orientation to the weighted circular mean 30 orientation $\bar{\theta}_{h}$ of the cluster, computed as

$$
\overline{\theta_{h}}=\arctan \left(\frac{S_{h}}{C_{h}}\right),
$$


where $S_{h}$ and $C_{h}$ correspond to

$$
\begin{aligned}
S_{h} & =\sum_{s_{i} \in \text { cluster } h} q_{i} \sin \left(2 \theta_{i}\right), \\
C_{h} & =\sum_{s_{i} \in \text { cluster } h} q_{i} \cos \left(2 \theta_{i}\right) .
\end{aligned}
$$

In Equations 14 and 15, $\theta_{i}$ is the orientation of segment $s_{i}$.

Formally,

$$
\alpha_{h}=\underset{s_{i} \in \text { cluster } h}{\operatorname{argmin}} D_{\text {ang }}\left(\overline{\theta_{h}}, \theta_{i}\right),
$$

where $D_{\text {ang }}$ gives the minimum angle between the orientations.

By selecting $\alpha_{h}$, the possible new pseudo-centroids for cluster $h$ are the points $l_{\alpha_{h}} \times l_{i}$, with $s_{i} \in$ cluster $h$. New seed $\beta_{h}$ is selected as the one that permits new pseudo-centroid $c_{h}$ to be the closest point to all other possible pseudo-centroids of the cluster. Formally,

$$
\beta_{h}=\underset{s_{i} \in \text { cluster } h}{\operatorname{argmin}} \sum_{s_{j} \in \text { cluster } h} D_{\text {proj }}\left(l_{\alpha_{h}} \times l_{j}, l_{\alpha_{h}} \times l_{i}\right) .
$$

Figure 5 illustrates a cluster $h$ with its new seeds $\alpha_{h}$ and $\beta_{h}$. The dashed segments are the candidates prior to the selection of $\beta_{h}$.

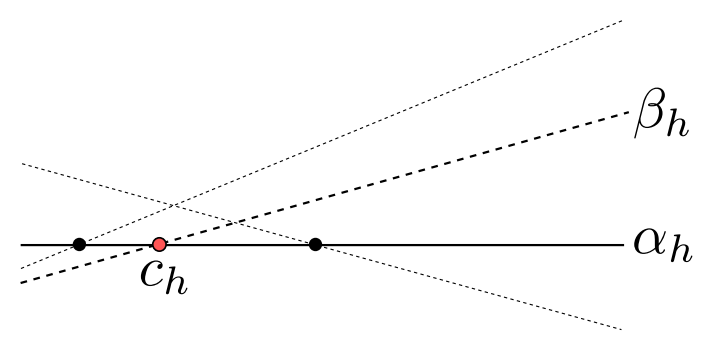

Figure 5: Illustration of a cluster $h$ with seeds $\alpha_{h}$ and $\beta_{h}$, and pseudo-centroid $c_{h}=l_{\alpha_{h}} \times l_{\beta_{h}}$. The dots represent all possible pseudo-centroids prior to the selection of $\beta_{h}$. The dashed segments are the candidates for seed $\beta_{h}$ prior to its selection. Seed $\beta_{h}$ is chosen based on the distance between the possible pseudo-centroids. tive steps. 


\subsection{Vanishing point detection}

After the convergence, the possible vanishing points associated with cluster $h$ are all intersection points in the cluster: $l_{i} \times l_{j}$, with $s_{i}, s_{j} \in$ cluster $h$. The vanishing point $v_{h}$ is the closest intersection to all segments in the cluster, i.e.,

$$
v_{h}=\underset{\substack{l_{i} \times l_{j} \\ s_{i}, s_{j} \in \text { cluster } h}}{\operatorname{argmin}} \sum_{s_{k} \in \text { cluster } h} D_{p r o j}\left(l_{k}, l_{i} \times l_{j}\right) .
$$

\section{Estimating vertical vanishing point and ground vanishing line}

According to [31], vertical vanishing points have two characteristics:

1. They usually are observed near the image y-axis, considering typical camera positions;

2. They are well separated from non-vertical vanishing points.

By considering these two characteristics as our premise, we can determine the vertical vanishing $v_{z}$, and the ground vanishing line $m$.

Regarding the first characteristic studied by [31], $v_{z}$ should be related to the cluster that has the closest mean orientation (Equation 13) to the y-axis of the image. But this condition alone does not suffice.

Figure 6 shows two possible clusters with their mean orientation represented by a dashed line. Note that the mean orientation in both clusters has no deviation from the y-axis. Nonetheless, a cluster associated with a vertical direction should have all segments with similar orientation, eliminating the possibility of choosing the first cluster.

Consequently, another characteristic that has to be analyzed is the distribution of the segments orientation in a cluster. For example, considering the segments orientation in Figure 6 the first cluster has greater standard deviation than the second cluster. This observation leads us to the second necessary condition for associating a cluster with the vertical direction: the segments orientation in the cluster must have low circular standard deviation [30, defined as

$$
\sigma_{h}=\frac{\sqrt{-2 \ln \left(\overline{R_{h}}\right)}}{2}
$$




\section{Cluster 1}

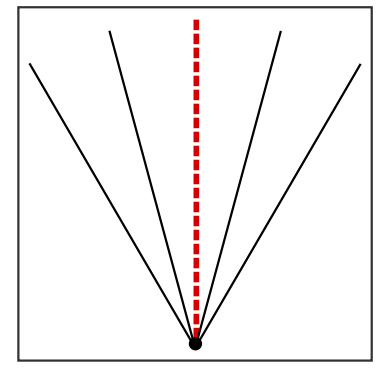

Cluster 2

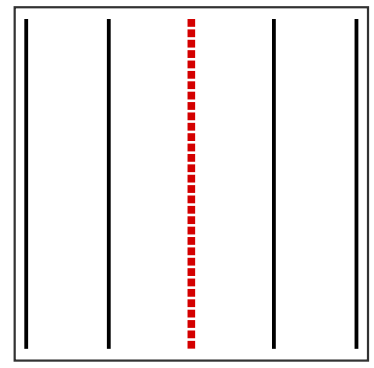

Figure 6: Illustration of two possible clusters. The mean orientation of each cluster is represented by a dashed line.

where $\overline{R_{h}}$ corresponds to

$$
\overline{R_{h}}=\frac{\sqrt{S_{h}^{2}+C_{h}^{2}}}{\sum_{s_{i} \in \text { cluster } h} q_{i}} .
$$

Combining these two conditions, we can select cluster $z$ associated with the vertical direction. Formally,

$$
z=\underset{h \in[1, H]}{\operatorname{argmin}} D_{a n g}\left(\overline{\theta_{h}}, \frac{\pi}{2}\right)+\sigma_{h}
$$

where $\overline{\theta_{h}}$ is the circular mean orientation of cluster $h$ (Equation 13 ) and $\sigma_{h}$ is the circular standard deviation of cluster $h$ (Equation 19).

Then vertical vanishing point $v_{z}$ is the one associated with cluster $z$.

To estimate the ground vanishing line $m$, we recall the second characteristic studied by [31]: all vanishing points are well separated from $v_{z}$. So, there are three cases to be considered, depending on the number of clusters chosen by the user:

- Two: the ground vanishing line is the one that contains the non-vertical vanishing point, and with orientation given by the mean orientation of its cluster.

- Three: the ground vanishing line is the one that connects the two nonvertical vanishing points. 
- Four or more: in this case, it is not clear through which two vanishing points the ground vanishing line passes. By graphically showing the possible vanishing lines, the user can select the one that corresponds to the ground plane.

\section{Experiments}

\footnotetext{
${ }^{2}$ The most orthogonal vanishing points are the ones with the lowest orthogonality error (Equation 25.
} 

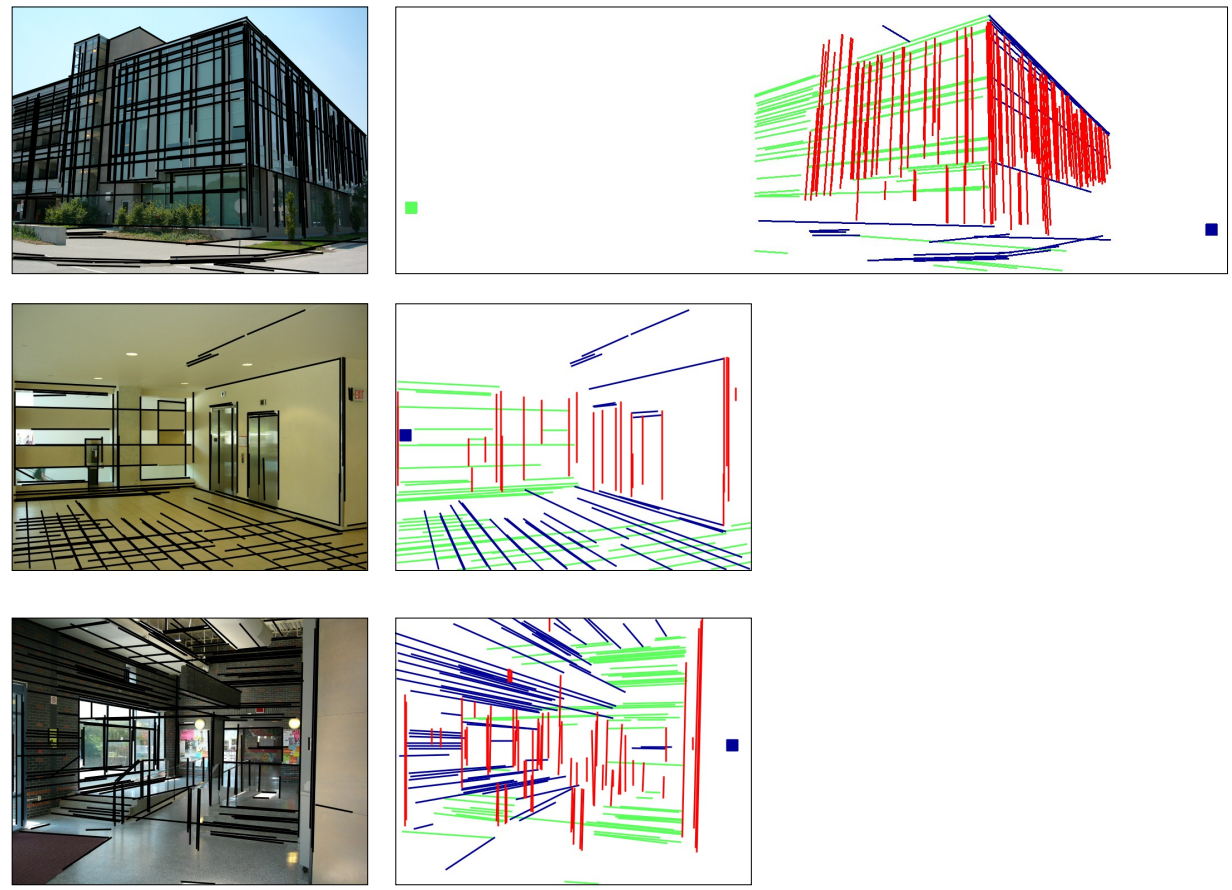

Figure 7: The first row presents three input images together with the detected segments in black. The second row shows the correspondent final segment clusters and colored squares representing the detected finite vanishing points. Red parallel segments represent a cluster associated with a infinite vanishing point.

intrinsic parameters, provided by the database. We compared the focal length calculated using the most orthogonal vanishing points to the real expected one.

\subsubsection{Orthogonality error}

The authors of the database provided the intrinsic parameters of the camera: focal length $f$, pixel dimension $\left(m_{x}, m_{y}\right)$, principal point $\left(p_{x}, p_{y}\right)$, and skew factor $\varsigma$. With this information, we can construct the camera intrinsic matrix:

$$
\mathrm{K}=\left[\begin{array}{ccc}
f / m_{x} & \varsigma & p_{x} \\
0 & f / m_{y} & p_{y} \\
0 & 0 & 1
\end{array}\right]
$$

Given that we have matrix K, it is trivial to obtain the Image of the Absolute 
Conic $\omega$ :

$$
\omega=\mathrm{K}^{-T} \mathrm{~K}^{-1}
$$

Considering two arbitrary orthogonal vanishing points $v_{p}$ and $v_{q}$, it is true that

$$
v_{p} \omega v_{q}=0
$$

Therefore, to find the most orthogonal vanishing points among all detected vanishing points $v_{i}, i=1, \ldots, M$, we select the triplet $\left(v_{p}, v_{q}, v_{r}\right)$ that minimizes the orthogonality error

$$
e_{p, q, r}=\left(v_{p} \omega v_{q}\right)^{2}+\left(v_{q} \omega v_{r}\right)^{2}+\left(v_{r} \omega v_{p}\right)^{2} .
$$

For each image on the York Urban database, we selected the most orthogonal vanishing point triplet. We then constructed a histogram of the cumulative orthogonality error for these triplets, shown in Figure 8 . We compared our method with the one provided in the database 32, named here as York Urban method, where the segments are hand detected and the computation is done by considering a cumulative space represented in a Gaussian Sphere.

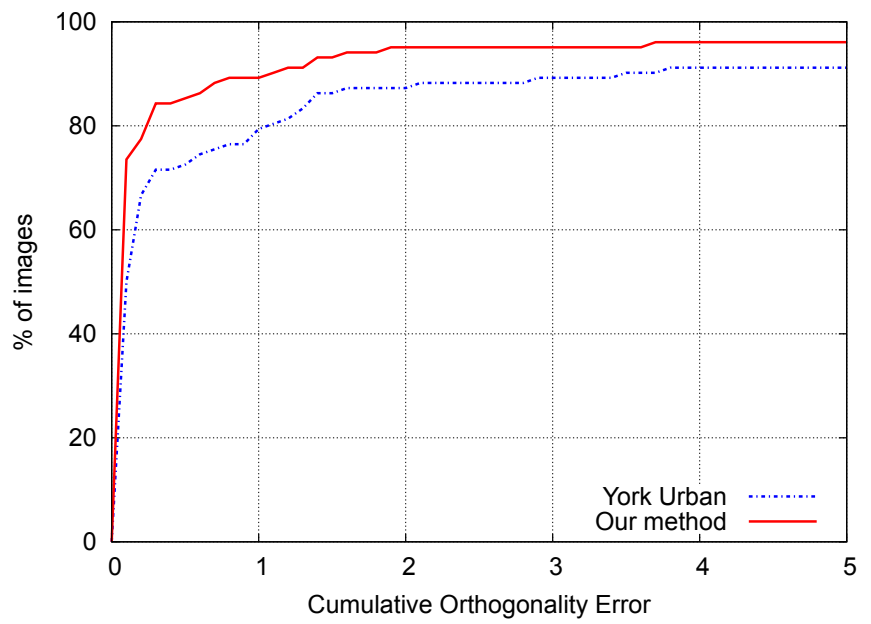

Figure 8: Orthogonality error cumulative histogram computed in the York Urban Database, with the most orthogonal vanishing point triplet in each image. Point $(x, y)$ represents $y \%$ of images with orthogonality error lower than $x$. 

cannot be done with images of scenes we don't have access to make reference measurements. Therefore we constructed a database of twenty images $(750 \times 563$ 


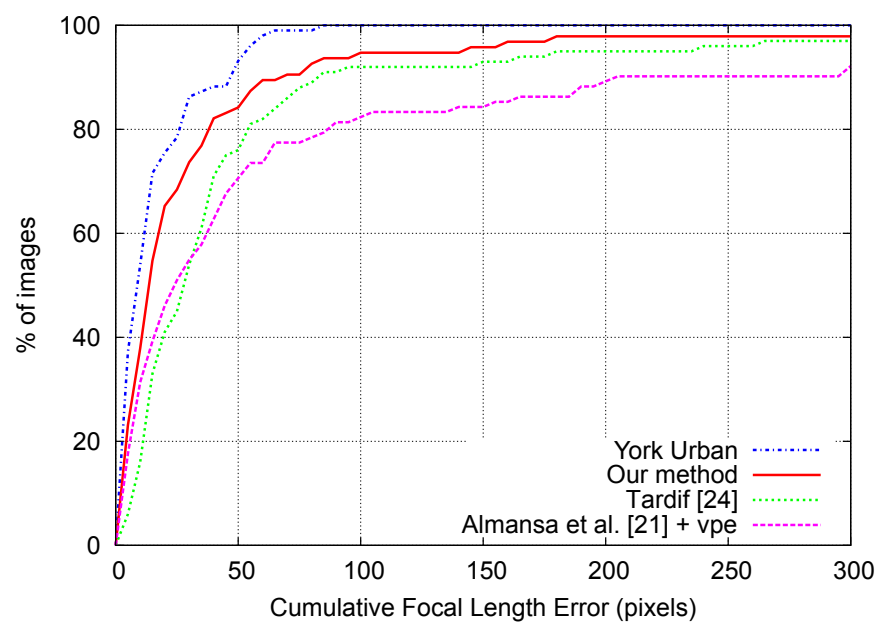

Figure 9: Focal length error cumulative histogram computed in the York Urban Database, with the most orthogonal vanishing point triplet in each image. Point $(x, y)$ represents $y \%$ of images with focal length error lower than $x$.

pixels) with reference objects in the real world. The vanishing points detected on these images would generate focal length erros of less than 25 pixels.

In order to measure the real height of an object $o b j$ depicted in the image, the user must inform its top and bottom points, $t_{o b j}$ and $b_{o b j}$, and the top and bottom points of a reference ref, $t_{r e f}$ and $b_{r e f}$, with known height $Z_{r e f}$.

In an error-free case, the points $t_{\alpha}$ and $b_{\alpha}, \alpha=o b j, r e f$, have to be aligned 315 with $v_{z}[6]$. Therefore, to compute height $Z_{o b j}$, we replace the location of the selected points $b_{o b j}$ and $b_{r e f}$ with their perpendicular projection onto lines $\overleftrightarrow{v_{z} t_{o b j}}$ and $\overleftrightarrow{v_{z} t_{r e f}}$, respectively, generating the scenario depicted in Figure 3

The errors associated with the choice of points $t_{o b j}, b_{o b j}, t_{r e f}$ and $b_{r e f}$ were considered by computing the height using all possible combinations of points inside a range of $1 \% \cdot\left|\overline{t_{\alpha} b_{\alpha}}\right|$ around each point, for $\alpha=o b j$, ref. For example, Figure 10 shows the considered rage around $t_{o b j}$ (top of object $o b j$ ). Each point inside the depicted range was considered once as $t_{o b j}$. The final height is the average between every obtained height.

The height of a person was measured comparatively to a known reference 


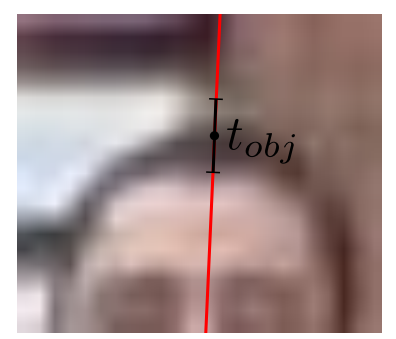

Figure 10: Range of $1 \% \cdot\left|\overline{t_{o b j} b_{o b j}}\right|$ around $t_{o b j}$.

325 is $171.5 \mathrm{~cm}$ and the mean observed error across all images was $\pm 0.58 \mathrm{~cm}$. Some of the results are shown in Figures 11, 12, and 13. In each one of these figures, the first column contains the input image with the segment clustering result, where each color represents a cluster associated with a vanishing point. The second column shows a zoomed crop with the vanishing line in green, the reference object height, and the computed height.

\section{Conclusion}

In this article, we described a method to detect vanishing points in an image and showed how to apply it to make efficient height measurements. The proposed method works with uncalibrated cameras, and can detect all vanishing points. Since it is performed in a bi-dimensional projective space, the detected points have accurate locations with no loss of information in transformations between spaces.

However, the method is only effective with images of man-made environments, in which it is possible to extract straight segments corresponding to different 3D orientations.

Several applications can benefit from the proposed method. In forensic investigations, for example, images from CCTV cameras - Closed-circuit television - can be used to estimate the height of suspects. CCTV cameras are widely 

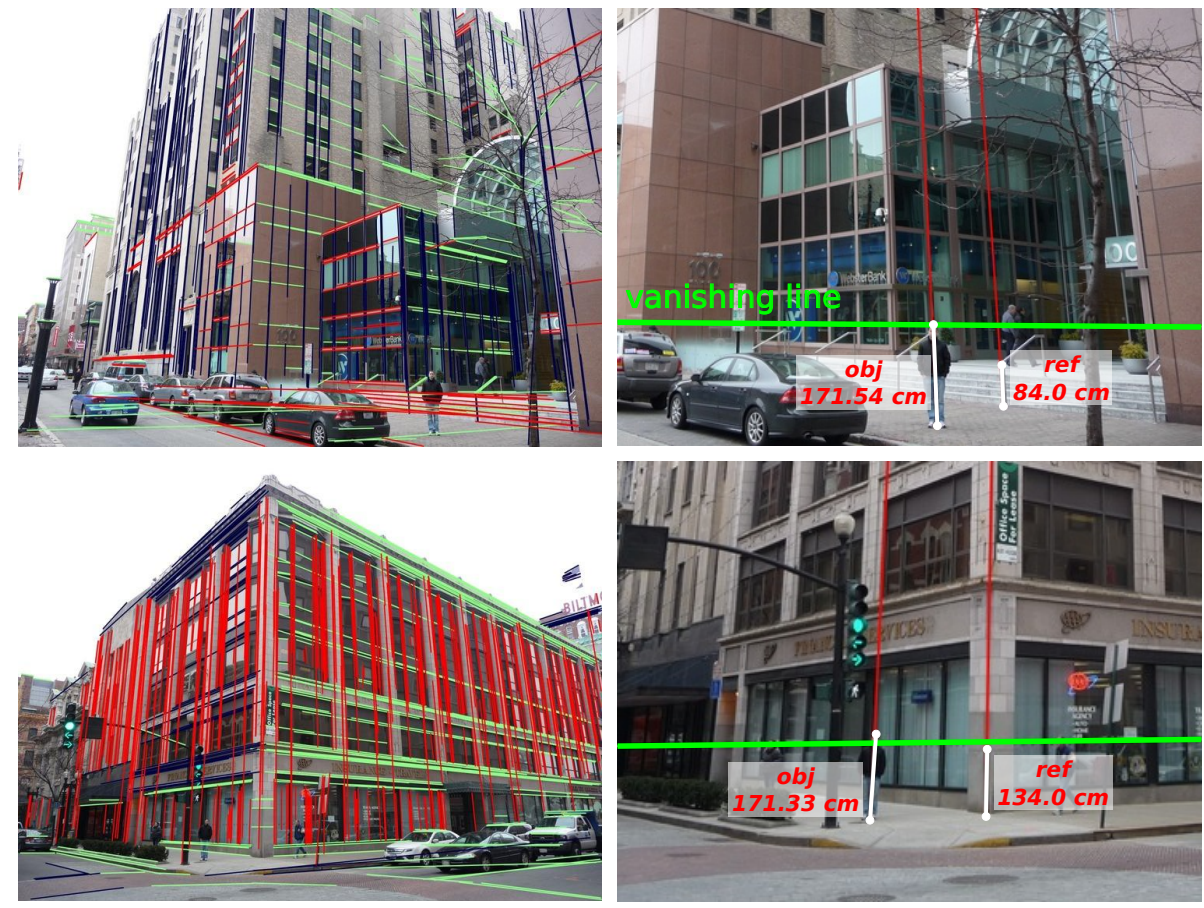

Figure 11: Measuring the height of a person. The first column contains the input image with the segment clustering result. The second column presents a crop with the computed height.

The results show visually and experimentally the effectiveness of the method and its application to a photogrammetry framework, allowing the estimation of heights in images.

The presented method is useful in computing the height of rigid objects or people standing in a straight position. However, the height of animated objects tend to change while they move. This study will be included in a future work.

\section{Acknowledgment}

This work was primarily supported by CNPq (grants 201238/2010-1 and 308882/2013-0), with additional support by FAPERJ and CAPES (grant E26/103.665/2012), NSF (grants IIS-0808718, CCF-0915661, and IIP-1330139), and FAPESP (grant 2012/50468-6). 

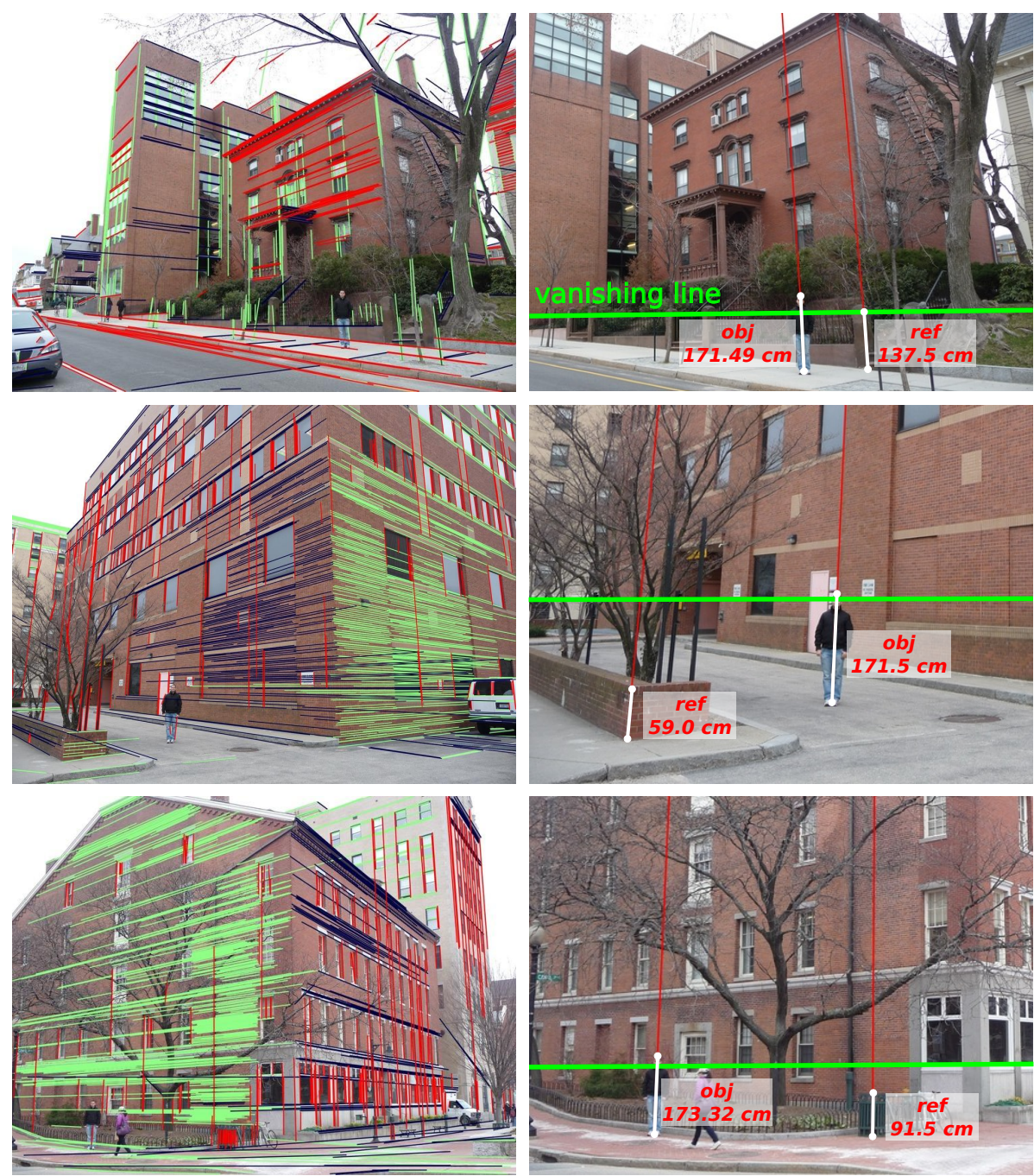

Figure 12: Measuring the height of a person. The first column contains the input image with the segment clustering result. The second column presents a crop the computed height.

\section{References}

[1] A. Saxena, M. Sun, A. Ng, Learning 3-D scene structure from a single still image, in: IEEE 11th International Conference on Computer Vision (ICCV), 2007, pp. 1-8.

[2] A. Saxena, M. Sun, A. Ng, Make3D: learning 3D scene structure from a 

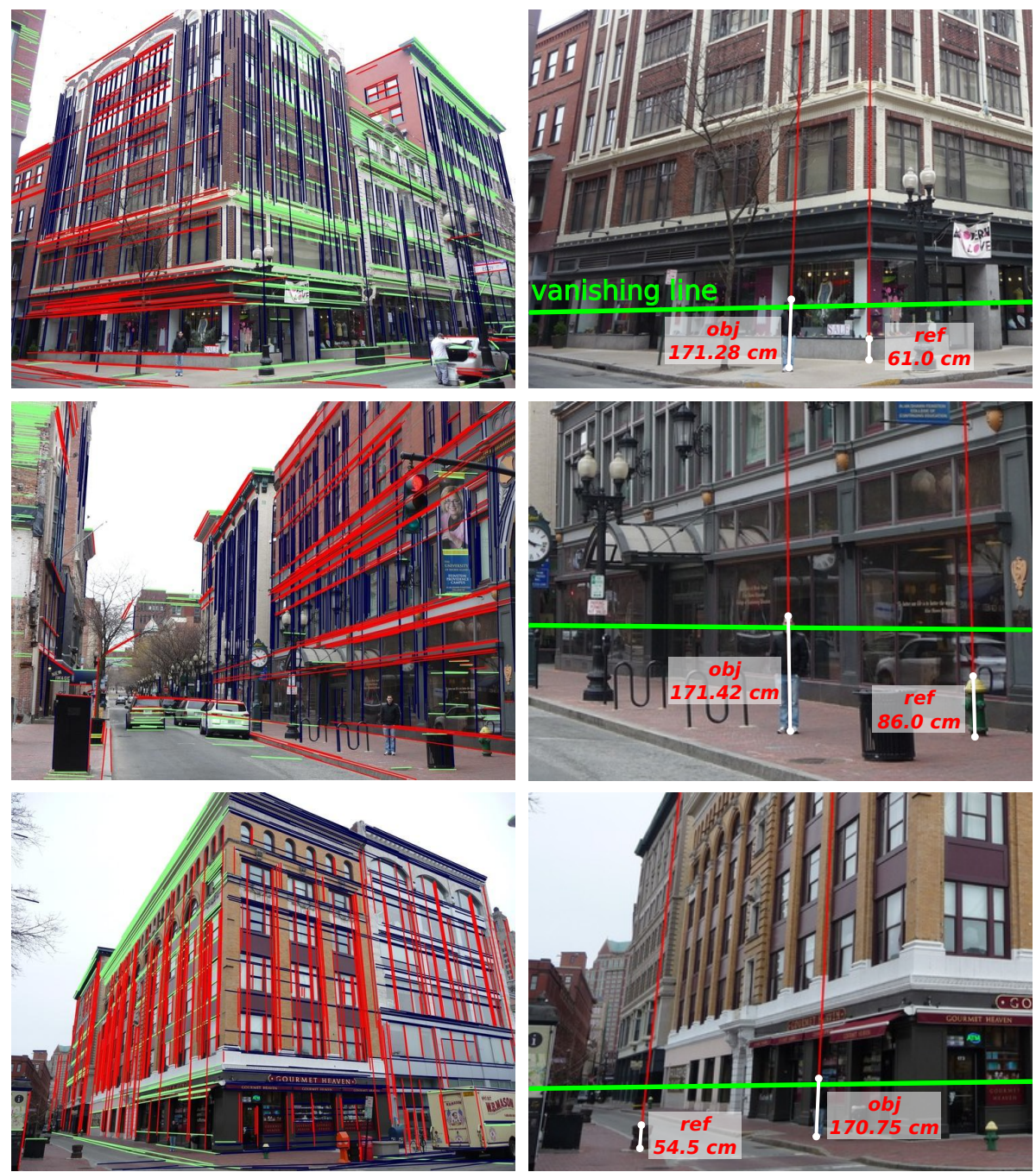

Figure 13: Measuring the height of a person. The first column contains the input image with the segment clustering result. The second column presents a crop the computed height.

single still image, IEEE Transactions on Pattern Analysis and Machine Intelligence 31 (5) (2009) 824-840.

[3] J. Michels, A. Saxena, A. Ng, High speed obstacle avoidance using monocular vision and reinforcement learning, in: Proceedings of the 22nd International Conference on Machine Learning (ICML), 2005, pp. 593-600. 
[4] J. Nascimento, J. Marques, Performance evaluation of object detection algorithms for video surveillance, IEEE Transactions on Multimedia 8 (4) (2006) 761-774.

[5] S. Bramble, D. Compton, L. Klasén, Forensic image analysis, in: 13th INTERPOL Forensic Science Symposium, 2001.

[6] A. Criminisi, Accurate visual metrology from single and multiple uncalibrated images, Springer-Verlag, New York, NY, USA, 2001.

[7] C. Madden, M. Piccardi, Height measurement as a session-based biometric for people matching across disjoint camera views, in: Image and Vision Computing New Zealand (IVCNZ), 2005, pp. 282-286.

[8] Z. Chen, N. Pears, B. Liang, A method of visual metrology from uncalibrated images, Pattern Recognition Letters 27 (13) (2006) 1447-1456.

[9] S. Khan, M. Shah, A multiview approach to tracking people in crowded scenes using a planar homography constraint, Proceedings of the 9th European Conference on Computer Vision (ECCV) (2006) 133-146.

[10] A. Criminisi, Single-view metrology: algorithms and applications, in: Proceedings of the 24th DAGM Symposium on Pattern Recognition, 2002, pp. 224-239.

[11] S.-W. Park, T.-E. Kim, J.-S. Choi, Robust estimation of heights of moving people using a single camera, in: Proceedings of the International Conference on IT Convergence and Security (ICITCS), 2011, pp. 389-405.

[12] K.-Z. Lee, A simple calibration approach to single view height estimation, in: Ninth Conference on Computer and Robot Vision (CRV), 2012, pp. $161-166$.

[13] N. Nguyen, R. Hartley, Height measurement for humans in motion using a camera: a comparison of different methods, in: International Conference 
on Digital Image Computing Techniques and Applications (DICTA), 2012, pp. 1-8.

[22] T. Tuytelaars, L. V. Gool, M. Proesmans, T. Moons, The cascaded Hough transform as an aid in aerial image interpretation, in: Sixth International Conference on Computer Vision (ICCV), 1998, pp. 67-72.

[23] J. Shufelt, Performance evaluation and analysis of vanishing point detec415

[24] A. Almansa, A. Desolneux, S. Vamech, Vanishing point detection without any a priori information, IEEE Transactions on Pattern Analysis and Machine Intelligence 25 (4) (2003) 502-507. 
[25] C. Rother, A new approach to vanishing point detection in architectural environments, Image and Vision Computing 20 (9) (2002) 647-655.

[26] G. McLean, D. Kotturi, Vanishing point detection by line clustering, IEEE Transactions on Pattern Analysis and Machine Intelligence 17 (11) (1995) 1090-1095.

[27] J.-P. Tardif, Non-iterative approach for fast and accurate vanishing point detection, in: IEEE 12th International Conference on Computer Vision (ICCV), 2009, pp. 1250-1257.

[28] A. Desolneux, L. Moisan, J.-M. Morel, Meaningful alignments, International Journal of Computer Vision 40 (1) (2000) 7-23.

[29] A. Desolneux, L. Moisan, J.-M. Morel, Maximal meaningful events and applications to image analysis, Annals of Statistics (2003) 1822-1851.

[30] K. Mardia, P. Jupp, Directional statistics, Wiley Series in Probability and Statistics, John Wiley and Sons, 1999.

[31] A. Gallagher, Using vanishing points to correct camera rotation in images, in: Proceedings of the 2nd Canadian Conference on Computer and Robot Vision, 2005, pp. 460-467.

[32] P. Denis, J. Elder, F. Estrada, Efficient edge-based methods for estimating manhattan frames in urban imagery, in: Proceedings of the 10th European Conference on Computer Vision (ECCV), 2008, pp. 197-210. 